\title{
Fatores preditivos de complicações graves em cirurgia neonatal
}

\section{Predictors of major postoperative complications in neonatal surgery}

\author{
Dora Catré ${ }^{1}$; Maria Francelina Lopes 2 ; Angel Madrigal 3 ; Bárbara Oliveiros ${ }^{4}$; António Silvério Cabrita ; $^{5}$ Joaquim Silva Viana ${ }^{6}$; \\ José FARELA NeVES ${ }^{7}$
}

\section{R E S U M O}

\begin{abstract}
Objetivo: investigar a incidência e gravidade das complicações pós-operatórias precoces e identificar fatores de risco para o seu desenvolvimento em recém-nascidos submetidos ao tratamento cirúrgico, sob anestesia geral. Métodos: análise retrospectiva dos dados de 437 neonatos com doença crítica submetidos à cirurgia neonatal num centro cirúrgico pediátrico terciário, entre janeiro de 2000 e dezembro de 2010. A gravidade das complicações ocorridas nos primeiros 30 dias de pós-operatório foi classificada utilizando o sistema de Clavien-Dindo para complicações cirúrgicas, sendo considerados graves os graus III a V. Por análise estatística uni e multivariada avaliaram-se variáveis pré e intraoperatórias com potencial preditivo de complicações pós-operatórias graves. Resultados: a incidência de, pelo menos, uma complicação grave foi $23 \%$, com uma mediana de uma complicação por paciente 1:3. Ao todo, ocorreram 121 complicações graves. Destas, 86 necessitaram de intervenção cirúrgica, endoscópica ou radiológica (grau III), 25 puseram em risco a vida, com disfunção uni ou multi-órgão (grau IV) e dez resultaram na morte do paciente (grau V). As principais complicações foram técnicas $(25 \%)$, gastrointestinais $(22 \%)$ e respiratórias (21\%). Foram identificados quatro fatores de risco independentes para complicações pós-operatórias graves: reoperação, operação por hérnia diafragmática congênita, prematuridade menor que 32 semanas de idade gestacional e cirurgia abdominal. Conclusão: a incidência de complicações pósoperatórias graves após cirurgias neonatais, sob anestesia geral, permaneceu elevada. As condições consideradas fatores de risco independentes para complicações graves após a cirurgia neonatal podem ajudar a definir o prognóstico pós-operatório em neonatos com doença cirúrgica e orientar as intervenções para melhoria de resultados.
\end{abstract}

Descritores: Morbidade. Cirurgia Geral. Índice de gravidade de doença. Avaliação de resultados (cuidados de saúde). Recémnascido.

\section{INTRODUÇÃO}

O tratamento cirúrgico neonatal evoluiu continuamente desde os últimos 50 anos. Apesar das melhorias nas técnicas anestésico/cirúrgicas, a cirurgia neonatal continua a ser uma causa importante de morbidade e mortalidade, especialmente na população altamente vulnerável de recém-nascidos ${ }^{1-3}$. Com uma melhor compreensão da gravidade e dos fatores de risco para o desenvolvimento de complicações pós-operatórias entre os recém-nascidos operados, os esforços podem ser direcionados para a prevenção da ocorrência de morbidade.

O sistema de classificação de complicações pósoperatórias apresentado por Clavien ${ }^{4}$, e revisado mais tar$\mathrm{de}^{5}$, foi recentemente utilizado em estudos pediátricos de várias áreas cirúrgicas. Esta classificação categoriza as complicações pós-operatórias desde o grau I a V, de acordo com a sua necessidade de tratamento.
A análise de fatores preditivos de complicações pós-operatórias em neonatos ainda é incompleta.

Ao aplicar a classificação de Clavien-Dindo ${ }^{5}$ a uma série cirúrgica de recém-nascidos em estado crítico, tratados em um centro regional pediátrico terciário, os objetivos do presente estudo foram, em primeiro lugar, analisar a incidência e gravidade das complicações pós-operatórias dentro dos primeiros 30 dias após procedimentos cirúrgicos neonatais, sob anestesia geral, no nosso centro e, em segundo lugar, investigar fatores de risco pré e intraoperatórios para o desenvolvimento de morbidade grave pós-operatória precoce.

\section{MÉTODOS}

Os dados para este estudo foram coletados a partir de uma base de dados mantida prospectivamente

Trabalho realizado na UTIP do Hospital Pediátrico de Coimbra (HPC).

1. Serviço de Anestesiologia, Centro Hospitalar Tondela-Viseu, EPE, Viseu, Portugal; 2. Serviço de Cirurgia Pediátrica, Centro Hospitalar e Universitário de Coimbra, EPE, Coimbra, Portugal; 3. Serviço de Anestesiologia Pediátrica, Centro Hospitalar e Universitário de Coimbra, EPE, Coimbra, Portugal; 4. Departamento de Bioestatítica e Informática Médica, Faculdade de Medicina, Universidade de Coimbra, Coimbra, Portugal; 5. Faculdade de Medicina, Universidade de Coimbra, Coimbra, Portugal; 6. Faculdade de Ciências da Saúde (Anestesiologia), Universidade da Beira Interior, Covilhã,Portugal; 7. Unidade de Cuidados Intensivos Pediátricos, Centro Hospitalar e Universitário de Coimbra, EPE, Coimbra, Portugal. 
pela unidade clínica de terapia intensiva pediátrica (UTIP), incluindo dados demográficos e dados clínicos de todos os recém-nascidos internados na UTIP do Hospital Pediátrico de Coimbra (HPC).

Analisaram-se retrospectivamente as complicações que ocorreram nos primeiros 30 dias de pós-operatório em 437 crianças, cuja cirurgia neonatal foi realizada no HPC entre janeiro de 2000 e dezembro de 2010.

Foram considerados casos elegíveis os que preencheram os seguintes critérios: pacientes recém-nascidos submetidos a procedimentos cirúrgicos, sob anestesia geral, durante o período neonatal (0-28 dias de vida), admitidos na UTIP, no pré-operatório ou nas primeiras seis horas pós-operatórias e cuja cirurgia foi concluída no HPC.

Excluíram-se os seguintes casos: 1) pacientes recém-nascidos submetidos à cirurgia fora do HPC; 2) pacientes recém-nascidos que foram submetidos à anestesia não associada a procedimentos cirúrgicos, 3) pacientes recém-nascidos operados no HPC mas sem admissão na UTIP.

A análise da base de dados clínicos foi completada com a revisão dos prontuários clínicos individuais. Foi obtida permissão institucional para rever a informação clínica dos pacientes, obedecendo às normas de proteção individual dos dados e aos requisitos éticos exigidos pelo Comitê de Ética em Pesquisa do nosso Centro Hospitalar. Foram coletadas características demográficas, informação clínica pré, intra e pós-operatórias e resultados.

Os resultados de interesse foram a identificação, quantificação e classificação de complicações pós-operatórias que ocorreram nos primeiros 30 dias após a cirurgia neonatal, utilizando a classificação de Clavien-Dindo ${ }^{5}$ (Tabela 1) e a identificação de fatores de risco pré e intraoperatórios para complicações pós-operatórias graves, definidas como complicações graus III a $\mathrm{V}$.

Complicações foram definidas como eventos adversos que não eram inerentes à condição pré-operatória cirúrgica.
Cirurgia abdominal foi definida como qualquer procedimento cirúrgico realizado na cavidade abdominal.

Os 437 pacientes foram distribuídos em dois grupos, com base nas complicações pós-operatórias. Um grupo incluiu pacientes com complicações Clavien-Dindo graus III a V (complicações graves). Os pacientes que não tiveram complicações ou que tiveram complicações de grau I ou Il foram utilizados como grupo de controle.

A associação potencial de risco de morbidade nos primeiros 30 dias de pós-operatório com características demográficas ao nascer e no ato cirúrgico, relacionadas ao procedimento operatório foram examinadas por análise univariada.

Algumas variáveis contínuas foram convertidas em variáveis categóricas para análise estatística. A análise do percentil de peso de nascimento foi realizada usando gráficos específicos para o sexo e idade de gestação ${ }^{6}$. Indicação cirúrgica por doença adquirida foi definida como condição cirúrgica que ocorreu após o nascimento.

As variáveis categóricas são apresentadas como valores absolutos (percentuais). As variáveis quantitativas são apresentadas como mediana e valores mínimo e máximo ou mediana e interquartil (percentis 25-75).

As variáveis quantitativas foram comparadas usando o teste de Mann-Whitney, conforme apropriado para a distribuição não normal dos dados. As variáveis qualitativas foram comparadas pelos testes $c^{2}$ e exato de Fisher, conforme apropriado.

Analisaram-se comparativamente as variáveis relacionadas com as características demográficas ao nascer e no ato cirúrgico e relacionadas com o procedimento operatório nos grupos com e sem complicações graves. As variáveis estatisticamente significativas $(p<0,05)$ foram selecionadas para inclusão em modelo de regressão logística múltipla, que foi utilizada para determinar os fatores preditivos independentes de complicações pós-operatórias graves. Assim, foram incluídas no nosso modelo

Tabela 1 - Classificação Clavien-Dindo de Complicações Cirúrgicas ${ }^{5}$.

\begin{tabular}{|c|c|}
\hline Grau & Definição \\
\hline । & $\begin{array}{l}\text { Qualquer desvio do curso pós-operatório normal sem necessidade de intervenção para além da administração de } \\
\text { antieméticos, antipiréticos, analgésicos, diuréticos, eletrólitos e fisioterapia \# }\end{array}$ \\
\hline ॥ & $\begin{array}{l}\text { Complicação requerendo tratamento farmacológico com outros medicamentos além dos que são permitidos para as } \\
\text { complicações de grau I. }\end{array}$ \\
\hline $\begin{array}{l}\| I I \\
I I I-\mathrm{a}\end{array}$ & $\begin{array}{l}\text { Complicação requerendo intervenção cirúrgica, endoscópica ou radiológica } \\
\text { Intervenção sem ser sob anestesia geral } \\
\text { Intervenção sob anestesia geral }\end{array}$ \\
\hline $\begin{array}{l}\text { IV } \\
\text { IV-a } \\
\text { IV-b }\end{array}$ & $\begin{array}{l}\text { Complicação com perigo de vida requerendo admissão em unidade de terapia intensiva } \\
\text { Disfunção de órgão único (incluindo diálise) } \\
\text { Disfunção multi-órgão }\end{array}$ \\
\hline V & Morte do paciente \\
\hline
\end{tabular}

"Este grau também inclui infeções cutâneas drenadas sem anestesia geral. 
as seguintes variáveis: nascimento prematuro com menos de 32 semanas de gestação, peso operatório na primeira cirurgia, mais do que uma intervenção anestésico/ cirúrgica, ASA 3 a 5, anestesia intravenosa, cirurgia abdominal e cirurgia de hérnia diafragmática congênita, enterocolite necrosante, defeitos congênitos da parede abdominal (onfalocele/gastrosquise), atresia intestinal e malformações anorretais. Foram realizados os testes de Hosmer-Lemeshow e Omnibus para determinar respectivamente a qualidade do ajuste e desempenho do modelo. Variáveis com odds ratio e intervalos de confiança de 95\% (IC) diferentes de um foram considerados como tendo uma associação significativa e independente com a morbidade.

Os níveis de significância $\mathrm{p}<0,05$ foram considerados estatisticamente significativos.

\section{RESULTADOS}

Dos 1055 recém-nascidos internados na UTIP entre 01 de janeiro de 2000 e 31 de dezembro de 2010, 437 (41\%) pacientes preencheram os critérios de inclusão. Nestes neonatos foram realizadas 558 intervenções sob anestesia geral, para um total de 636 procedimentos cirúrgicos.

A média de idade gestacional das 437 crianças ao nascer foi 37 semanas (variação, 24-41) e a mediana do peso de nascimento foi 2760 gramas (variação, 4404350). A análise estatística da idade gestacional e do peso, ao nascer, não apresentaram diferença significativa entre os grupos com e sem complicações graves.

No total, 56\% (244) dos recém-nascidos eram do sexo masculino, $15 \%$ (66) nasceram prematuros com menos de 32 semanas de gestação, dos quais 90\% (56) eram de muito baixo peso (menos de 1500 gramas), e 81,5\% (356) apresentaram malformações congênitas que necessitaram de cirurgia neonatal.

Dos 437 recém-nascidos submetidos a procedimentos anestésicos/cirúrgicos, 242 (55\%) não apresentaram nenhuma complicação pós-operatória. Trezentas e setenta complicações foram registradas até ao $30^{\circ}$ dia de pós-operatório, num total de 195 crianças, das quais, 99
(23\%) apresentaram 121 complicações graves (intervalo, 1-3, mediana 1).

As complicações categorizadas de acordo com a classificação de Clavien-Dindo são apresentadas na tabela 2, e o tipo e tempo de ocorrência de complicações são apresentados na tabela 3. Dois terços de todas as complicações foram de grau I ou ll e foram, na sua maioria, hematológicas, metabólicas e infecciosas. Das 121 complicações graves, 86 necessitaram de intervenção cirúrgica, endoscópica ou radiológica (grau III), 25 eram potencialmente fatais, com disfunção uni ou multi-órgão (grau IV) e dez resultaram na morte do paciente (grau V). As principais complicações foram predominantemente técnicas (25\%), gastrointestinais (22\%) e respiratórias (21\%). Dentre todas as complicações pós-operatórias, cerca de metade (51\%) foram identificadas nas primeiras 48 horas, $37 \%$ entre o segundo e o nono dias e os restantes $12 \%$ entre o décimo e o $30^{\circ}$ dias.

As complicações graves de grau $\mathrm{V}$ foram responsáveis por $45 \%$ (10/22) das mortes nos primeiros 30 dias de pós-operatório.

O efeito das variáveis pré e intraoperatórias sobre a ocorrência e gravidade das complicações pós-operatórias é apresentado na tabela 4. A ocorrência de complicações graves (graus III a V) foi significativamente associada a parto prematuro com menos de 32 semanas de idade gestacional $(p=0,024)$ e a um peso operatório sig-

Tabela 2 - Categorização de 370 complicações baseada no sistema de classificação de Clavien-Dindo.

\begin{tabular}{lrr}
\hline Categorização/Grau & $\mathrm{n}(\%)$ \\
\hline Complicações menores & 36 & $(9,7)$ \\
Grau I & 213 & $(57,6)$ \\
Grau II & & \\
Complicações graves & 22 & $(5,9)$ \\
Graullla & 64 & $(17,3)$ \\
Graulllb & 13 & $(3,5)$ \\
GraulVa & 12 & $(3,2)$ \\
GraulVb & 10 & $(2,7)$ \\
Grau V & & \\
\hline
\end{tabular}

Tabela 3 - Tipo e tempo de ocorrência das complicações.

\begin{tabular}{lcccc}
\hline Tipo de complicação & Grave $(\mathrm{n}=\mathbf{1 2 1}), \%$ & Menor $(\mathrm{n}=\mathbf{2 4 9 ) ,} \%$ & Tempo - dias, mediana (variação) \\
\hline Cardiovascular & 12,4 & 11,6 & 0 & $(0-17)$ \\
Respiratória & 20,7 & 6,8 & $2,5(0-25)$ \\
Gastrointestinal & 22,3 & 1,6 & 6 & $(0-28)$ \\
Renal & 1,6 & 3,2 & 1 & $(0-5)$ \\
Neurológica & 5,8 & 2,8 & 5 & $(0-24)$ \\
Metabólica & 0 & 21,7 & 0 & $(0-23)$ \\
Hematológica & 0,8 & 29,3 & 0 & $(0-15)$ \\
Infecciosa & 11,6 & 18,5 & 6 & $(0-25)$ \\
Técnica & 24,8 & 4,4 & 6 & $(0-17)$ \\
\hline
\end{tabular}


nificativamente menor na primeira operação $(p=0,046)$. As crianças com mais de uma intervenção anestésico/ cirúrgica $(p<0,001)$, classificação ASA 3 a $5(P<0,001)$, anestesia intravenosa $(P=0,015)$, reparação cirúrgica de hérnia diafragmática congênita $(p=0,004)$, defeitos congênitos da parede $(p=0,033)$, enterocolite necrosante $(p=0,008)$, atresia do intestino delgado $(p=0,040)$, malformação anorretal $(p=0,030)$ e cirurgia abdominal $(p<0,001)$ foram significativamente mais propensas a apresentar complicações graves. Por outro lado, as restantes variáveis da tabela 4, também testadas com estatística univariada, não foram significativamente associadas à complicações graves.

Com base na análise univariada foram incluídas as seguintes variáveis no nosso modelo de regressão logística multivariada para determinar os fatores preditivos das complicações pós-operatórias graves: nascimento prematuro com menos de 32 semanas de gestação, peso operatório na primeira cirurgia, mais do que uma intervenção anestésico/cirúrgica, ASA 3 a 5, anestesia intravenosa, cirurgia abdominal e cirurgia de hérnia diafragmática congênita, enterocolite necrosante, defeitos congênitos da

Tabela 4 - Características dos neonatos que apresentaram complicações graves (graus de Clavien-Dindo e " III) e dos que tiveram complicações de Clavien-Dindo graus I ou II e sua associação com complicações dentro de 30 dias após a operação na análise univariada.

\begin{tabular}{|c|c|c|c|}
\hline Fatores & Complicações Clavien-Dindo & $\begin{array}{l}\text { Clavien-Dindo } \\
\text { Sem ou < II, n=338 }\end{array}$ & $P$ \\
\hline \multicolumn{4}{|l|}{ Características do paciente ao nascer } \\
\hline Gênero masculino, $n=244$ & 56 & 188 & 0,868 \\
\hline Prematuro<32 s IG, n=66 & 22 & 44 & 0,024 * \\
\hline PIG (<percentil10), n=67 & 18 & 49 & 0,371 \\
\hline GIG (>percentil 90), n=17 & 5 & 12 & 0,497 \\
\hline Apgar aos $5 \min <7, n=16^{\ddagger}$ & $5 / 97$ & $11 / 331$ & 0,403 \\
\hline >1 malformação congênita, $n=91$ & 20 & 71 & 0,862 \\
\hline \multicolumn{4}{|c|}{ Características do paciente no ato operatório } \\
\hline Idade na $1^{\text {a }}$ operação, dias de vida\# & $2(0-8)$ & $3(1-9)$ & 0,256 \\
\hline Peso na $1^{\text {a }}$ operação, $\mathrm{Kg}{ }^{\#}$ & $2,6(1,8-3)$ & $2,8(2-3,3)$ & 0,026 * \\
\hline >1 intervenção anestésica/cirúrgica, n=95 & 56 & 39 & $<0,001$ * \\
\hline \multicolumn{4}{|c|}{ Características operatórias (em pelo menos um procedimento por paciente) } \\
\hline Indicação cirúrgica adquirida, n=99 & 22 & 77 & 0,907 \\
\hline Score ASA e" 3, n=207 & 67 & 140 & $<0,001$ * \\
\hline Anestesia balanceada, $\mathrm{n}=372$ & 85 & 287 & 0,816 \\
\hline Anestesia intravenosa, $\mathrm{n}=75$ & 25 & 50 & $0,015^{*}$ \\
\hline Anestesia inalatória, $\mathrm{n}=8$ & 1 & 7 & 0,689 \\
\hline Cirurgia para:Atresia do esófago, $n=42$ & 8 & 34 & 0,557 \\
\hline Defeitos de parede abdominal, $n=49$ & 17 & 32 & $0,033 *$ \\
\hline Hérnia diafragmática congênita, $n=42$ & 17 & 25 & 0,004 * \\
\hline Enterocolite necrosante, $n=31$ & 13 & 18 & 0,008 * \\
\hline Obstrução duodenal, n=25 & 8 & 17 & 0,250 \\
\hline Atresia intestino delgado, $n=16$ & 7 & 9 & 0,040 * \\
\hline Ileus meconial, $n=9$ & 2 & 7 & 1,00 \\
\hline Malformações anorretais, $n=30$ & 2 & 28 & 0,030 * \\
\hline Doença de Hirschsprung, $n=6$ & 3 & 3 & 0,133 \\
\hline Hidronefrose congênita, $n=4$ & 2 & 2 & 0,222 \\
\hline Malformações cardíacas, n=31 & 6 & 25 & 0,649 \\
\hline Hidrocefalia adquirida, $n=30$ & 8 & 22 & 0,586 \\
\hline Mielomeningocelo, $n=27$ & 3 & 24 & 0,139 \\
\hline Cirurgia abdominal, $n=225$ & 70 & 155 & $<0,001$ * \\
\hline Cirurgia torácica, n=62 & 12 & 50 & 0,503 \\
\hline Duração da cirurgia $>2 \mathrm{~h}, \mathrm{n}=177$ & 47 & 130 & 0,108 \\
\hline
\end{tabular}

$n$, $n^{\circ}$ de casos; \#, Mediana e interquartil; s, semanas; IG, idade de gestação; $¥$, nove casos com valores de Apgar em falta foram excluídos da análise desta variável; PIG, pequeno para a idade de gestação; GIG, grande para a idade de gestação; min, minutos; >1, mais do que um; score ASA, estado físico estratificado pelo sistema de classificação Associação Americana de Anestesiologistas; *, estatisticamente significativo. 
parede abdominal (onfalocele/gastrosquise), atresia intestinal e malformações anorretais. O nosso modelo apresentou bom desempenho e boa adequação, respectivamente pelo teste Omnibus $\left(c^{2}=111,853, P<0,001\right)$ e pelo teste Hosmer-Lemeshow $\left(c^{2}=0,347, p=0,987\right)$.

Apenas quatro fatores mantiveram significância estatística na análise multivariada (Tabela 5): mais do que uma intervenção, reparação cirúrgica de hérnia diafragmática congênita, prematuridade com menos de 32 semanas de gestação e cirurgia abdominal.

\section{DISCUSSÃO}

O presente estudo tem dois achados principais. Em primeiro lugar, as crianças com procedimentos anestésico/ cirúrgicos neonatais tiveram uma incidência alta $(23 \%)$ de complicações pós-operatórias graves (complicações graus III a $\checkmark$ da classificação de Clavien-Dindo). Em segundo lugar, foram encontrados quatro fatores de risco independentes para complicações pós-operatórias graves em recém-nascidos operados: mais do que uma intervenção anestésico/cirúrgica, cirurgia de hérnia diafragmática congênita, prematuridade com menos de 32 semanas de gestação e cirurgia abdominal.

A UTIP do nosso hospital é uma unidade regional de cuidados terciários para cerca de 100 pacientes recém-nascidos por ano, incluindo todos os casos cirúrgicos. A região central de Portugal é servida por outros dois Centros perinatais terciários afiliados com a nossa UTIP médico-cirúrgica e por um serviço especializado de transporte neonatal e pediátrico de emergência para transferir os recém-nascidos em risco de outras unidades de saúde dentro da nossa área de referência. No HPC a equipe de prestadores de cuidados de saúde é altamente especializada no tratamento de neonatos com a vida em risco, incluindo cirurgiões e anestesiologistas pediátricos. Todas as especialidades de cirurgia neonatal estão disponíveis neste hospital, exceto para cirurgia cardíaca de coração aberto que é realizada num hospital afiliado de adultos.

Este estudo mostra que a cirurgia neonatal continua a ser uma causa importante de morbidade grave, mesmo num centro de referência para esta cirurgia. Um dos objetivos do estudo foi quantificar a incidência de várias complica- ções de procedimentos anestésico/cirúrgicos neonatais, proporcionando aos médicos da unidade de terapia intensiva, cirurgiões e anestesistas, a informação necessária ao devido esclarecimento dos pais sobre os riscos da cirurgia neonatal.

Este estudo é o primeiro a determinar a taxa de complicações pós-operatórias graves na sequência de uma ampla variedade de procedimentos cirúrgicos neonatais. Além disso, permitiu a sistematização e categorização por graus de gravidade através do uso de um sistema de classificação cirúrgica conhecido para categorização de complicações pósoperatórias em Cirurgia Geral. Desde 2004 essa classificação foi usada em múltiplos estudos de adultos e em vários estudos pediátricos, confirmando ser uma ferramenta confiável para medir os resultados em saúde ${ }^{7,8}$. No entanto, que seja do nosso conhecimento, embora tenham sido publicados recentemente muitos estudos sobre o assunto utilizando esta classificação ${ }^{7}$, existem poucos em Cirurgia Pediátrica ${ }^{9-11}$ e nenhum referente ao período neonatal.

Uma dimensão adicional deste estudo foi o uso sistemático deste sistema de classificação para caracterizar a gravidade das complicações numa série de neonatos operados, em que todos compartilhavam as características comuns de terem sido operados, sob anestesia geral, para tratamento de uma grande variedade de problemas clínicos no período neonatal e estadia pós-operatória na UTIP. No estudo atual foi fornecido detalhe suficiente pelo sistema de classificação de Clavien-Dindo, tendo-se estimado uma taxa de $23 \%$ de complicações graves, incluindo uma taxa de $2,7 \%$ de complicações de grau $V$. Além disso, este sistema de classificação foi fácil de usar.

O progresso em cirurgia neonatal alcançando um elevado grau de sucesso e relatos cada vez mais baixos de resultados adversos foi negativamente influenciado por diversos fatores demográficos que se sabe estarem relacionados com maior risco de complicações pós-operatórias graves, e que são muito frequentes nesta população. A prematuridade e baixo peso ao nascer são exemplos desses fatores ${ }^{12-16}$. Além do fato de estas condições serem muitas vezes associadas a um processo estressante para os componentes da equipe de saúde no bloco operatório, mesmo para os mais experientes, esses neonatos são, em geral, doentes graves, criando um ambiente hostil com maior propensão para complicações.

Tabela 5 - Fatores preditivos independentes para complicações pós-operatórias graves precoces entre os neonatos cirúrgicos admitidos na UTIP.

\begin{tabular}{lcccr}
\hline Fator & \multicolumn{3}{c}{ Complicação pós-operatória grave precoce } \\
\cline { 2 - 5 } & Odds ratio & $95 \%$ & IC & $P$ \\
\hline Mais do que uma intervenção & 12,008 & 6,795 & 21,223 & $<0,001$ \\
Reparação de HDC & 3,843 & 1,732 & 8,526 & 0,001 \\
Prematuro <32 s IG & 2,666 & 1,355 & 5,245 & 0,005 \\
Cirurgia abdominal & 2,541 & 1,462 & 4,416 & 0,001 \\
\hline
\end{tabular}

Fonte: UTIP indica unidade de terapia intensiva pediátrica; HDC, hérnia diafragmática congênita; <32 s IG, menor que 32 semanas de idade de gestação; IC, intervalo de confiança. 
Em concordância com outros estudos que analisaram os resultados pós-operatórios em bebês prematuros em contextos cirúrgicos específicos ${ }^{12,15}$, o nascimento prematuro com menos de 32 semanas de gestação influenciou significativamente um resultado adverso no pós-operatório de cirurgia neonatal nas análises estatísticas uni e multivariada. No estudo atual, esta característica aumentou em 2,7 vezes a probabilidade de ter uma complicação pós-operatória grave.

Entre os vários problemas clínicos enfrentados pela população cirúrgica neonatal, os neonatos submetidos à cirurgia abdominal para tratamento de uma grande variedade de condições foram significativamente mais propensos a apresentar importantes complicações pós-operatórias. Destes, podemos especificar o tratamento cirúrgico para enterocolite necrosante ${ }^{17}$, uma doença conhecida pelo risco à vida que representa, principalmente relacionada à imaturidade e baixo peso ao nascer ${ }^{17-19}$, e tratamento cirúrgico para várias doenças congênitas ${ }^{20-23}$, ou seja, para onfalocele/gastrosquise ${ }^{20}$ e atresia intestinal ${ }^{21}$. Enquanto todos estes fatores perderam significado estatístico no nosso modelo multivariado, a cirurgia para a correção do defeito diafragmático congênito foi um forte fator preditivo de complicações graves. No nosso estudo, as crianças que precisaram de reparação de defeitos diafragmáticos congênitos apresentaram significativamente maior probabilidade de complicações pós-operatórias. Neste e em outros estudos ${ }^{22-23}$, a reparação de defeitos diafragmáticos foi realizada por via abdominal ou torácica e notou-se que a abordagem cirúrgica por via abdominal levou frequentemente à complicações gastrointestinais que necessitaram de reoperação abdominal22.

Apesar do nosso esforço, ocorreram algumas limitações neste estudo. O projeto foi retrospectivo, podendo introduzir vieses na classificação. Além disso, os dados utilizados referem-se a um único centro, e a população do estudo é limitada aos pacientes internados na nossa unidade de atendimento intensivo terciário, não considerando, por um lado, a maior morbidade específica das crianças operadas fora do nosso hospital pediátrico (procedimentos de coração aberto) e, por outro lado, a menor morbidade dos recém-nascidos operados sem doença crítica ou fatores de risco suficientemente graves para justificar a admissão em unidade de terapia intensiva. Além disso, embora este estudo inclua um bom número de pacientes, relata o registro da gravidade das complicações ocorridas no pósoperatório em toda a cirurgia neonatal, que é muito ampla, que inclui afecções e malformações congênitas com diferentes prognósticos e complicações.

Estas limitações são superadas pelos pontos fortes deste estudo. Em primeiro lugar, preenche uma lacuna na literatura abordando especificamente as complicações da população cirúrgica neonatal, fazendo-o em termos de quantificação e sistematização em categorias e também pelo estabelecimento de associações com fatores de risco. Em segundo lugar, foi baseado numa base de dados muito completa, que incluiu detalhes demográficos e clínicos de uma grande amostra de pacientes cirúrgicos neonatais de uma vasta área do nosso país coletados por mais de uma década. A novidade da utilização do sistema de ClavienDindo à cirurgia neonatal e sua aparente utilidade na avaliação dos resultados nesta população é, no entanto, contrabalançada pela necessidade de novos estudos para avaliar essa classificação de acordo com as diferentes malformações e afecções do período neonatal.

Em resumo, o presente estudo apresenta, pela primeira vez, uma ampla análise sistematizada de complicações pós-operatórias numa grande variedade de procedimentos cirúrgicos neonatais. Além disso, é o único estudo, de que temos conhecimento, que fornece informações sobre incidência e fatores preditivos de morbidade grave na população neonatal cirúrgica. As condições consideradas fatores de risco independentes para complicações graves após a cirurgia neonatal podem ajudar a definir o prognóstico pós-operatório em neonatos com doença cirúrgica e orientar as intervenções para melhoria de resultados. O reconhecimento dos fatores de mau prognóstico permite aconselhamento informado das famílias e previsão mais precisa dos eventuais resultados.

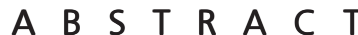

\begin{abstract}
Objective: To investigate the incidence and severity of early postoperative complications and to identify their risk factors in newborns undergoing surgery under general anesthesia. Methods: We conducted a retrospective analysis of data from 437 critically ill newborns undergoing surgery in a tertiary pediatric surgical center, between January 2000 and December 2010. Complications that occurred within the first 30 days after surgery were classified using the Clavien-Dindo system, for which grades III to $\mathrm{V}$ were considered severe. We used univariate and multivariate analysis to evaluate pre- and intraoperative variables potentially predictive of severe postoperative complications. Results: The incidence of at least one serious complication was $23 \%$, with a median of one complication per patient 1:3. Altogether, there were 121 serious complications. Of these, 86 required surgical, endoscopic or radiological interventions (grade III), 25 endangered life, with uni or multi-organ failure (grade IV) and ten resulted in death (grade V). The most common complications were technical (25\%), gastrointestinal (22\%) and respiratory (21\%). We identified four independent risk factors for severe postoperative complications: reoperation, operation for congenital diaphragmatic hernia, preterm birth less than 32 weeks of gestational age and abdominal surgery. Conclusion: The incidence of severe postoperative complications after neonatal surgeries under general anesthesia remains high. The conditions considered independent risk factors for those can guide interventions to improve results.
\end{abstract}

Key words: Morbidity. General surgery. Severity of illness index. Outcome assessment (health care). Infant, newborn. 


\section{REFERÊNCIAS}

1. Horbar JD, Carpenter JH, Badger GJ, Kenny MJ, Soll RF, Morrow $\mathrm{KA}$, et al. Mortality and neonatal morbidity among infants 501 to 1500 grams from 2000 to 2009. Pediatrics. 2012;129(6):1019-26.

2. Tomé T, Guimarães H, Bettencourt A, Peixoto JC. Neonatal morbimortality in very low birth weight in Europe: the Portuguese experience. J Matern Fetal Neonatal Med. 2009;22 Suppl 3:85-7.

3. Neto MT, Tavares MN, Serelha M, Casella P. A vertente cirúrgica de uma unidade de cuidados intensivos neonatais - 25 anos de experiência. Acta Pediatr Port. 2010;41(6):241-5.

4. Clavien PA, Sanabria JR, Strasberg SM. Proposed classification of complications of surgery with examples of utility in cholecystectomy. Surgery. 1992;111(5):518-26.

5. Dindo D, Demartines N, Clavien PA. Classification of surgical complications: a new proposal with evaluation in a cohort of 6336 patients and results of a survey. Ann Surg. 2004;240(2):205-13.

6. Olsen IE, Groveman SA, Lawson ML, Clark RH, Zemel BS. New intrauterine growth curves based on United States data. Pediatrics. 2010;125(2):214-24

7. Clavien PA, Barkun J, de Oliveira ML, Vauthey JN, Dindo D, Schulick $\mathrm{RD}$, et al. The Clavien-Dindo classification of surgical complications: five-year experience. Ann Surg. 2009;250(2):187-96.

8. Slankamenac K, Graf R, Puhan MA, Clavien PA. Perception of surgical complications among patients, nurses and physicians: a prospective cross-sectional survey. Patient Saf Surg. 2011;22;5(1):30.

9. Freilich DA, Cilento BG Jr, Graham D, Zhou J, Retik AB, Nguyen HT. Perioperative risk factors for surgical complications in pediatric urology: a pilot study in preoperative risk assessment in children. Urology. 2010;76(1):3-8.

10. Weinberg AC, Huang L, Jiang $H$, Tinloy $B$, Raskas MD, Penna FJ, et al. Perioperative risk factors for major complications in pediatric surgery: a study in surgical risk assessment for children. J Am Coll Surg. 2011:212(5):768-78.

11. Harraz AM, Shokeir AA, Soliman SA, El-Hefnawy AS, Kamal MM, Kamal Al, et al. Toward a standardized system for reporting surgical outcome of pediatric and adolescent live donor renal allotransplantation. J Urol. 2012;187(3):1041-6.

12. Blume ED, Altmann K, Mayer JE, Colan SD, Gauvreau K, Geva T. Evolution of risk factors influencing early mortality of the arterial switch operation. J Am Coll Cardiol. 1999;33(6):1702-9.

13. Tsao K, Allison ND, Harting MT, Lally PA, Lally KP. Congenital diaphragmatic hernia in the preterm infant. Surgery. 2010;148(2):404-10
14. Lai HS, Lin WH, Chen HA, Fan SZ, Chou HC. Successful laparotomy in a $432 \mathrm{~g}$ extremelylow-birth-weight infant with focal intestinal perforations. Int Surg. 2010;95(1):57-9.

15. Knottenbelt G, Costi D, Stephens P, Beringer R, Davidson A. An audit of anesthetic management and complications of tracheoesophageal fistula and esophageal atresia repair. Paediatr Anaesth. 2012:22(3):268-74.

16. Spitz L. Oesophageal atresia. Orphanet J Rare Dis. 2007;11;2:24

17. Thyoka M, de Coppi P, Eaton S, Khoo K, Hall NJ, Curry J, et al. Advanced necrotizing enterocolitis part 1: mortality. Eur J Pediatr Surg. 2012;22(1):8-12

18. Gephart SM, McGrath JM, Effken JA, Halpern MD. Necrotizing enterocolitis risk: state of the science. Adv Neonatal Care. 2012;12(2):77-87.

19. Eicher C, Seitz G, Bevot A, Moll M, Goelz R, Arand J, et al. Surgical management of extremely low birth weight infants with neonatal bowel perforation: a single-center experience and a review of the literature. Neonatology. 2012;101(4):285-92.

20. Christison-Lagay ER, Kelleher CM, Langer JC. Neonatal abdominal wall defects. Semin Fetal Neonatal Med. 2011;16(3):164-72.

21. Burjonrappa S, Crete E, Bouchard S. Comparative outcomes in intestinal atresia: a clinical outcome and pathophysiology analysis Pediatr Surg Int. 2011;27(4):437-42.

22. St Peter SD, Valusek PA, Tsao K, Holcomb GW 3rd, Ostlie DJ, Snyder CL. Abdominal complications related to type of repair for congenital diaphragmatic hernia. J Surg Res. 2007;140(2):234-6.

23. Safavi A, Synnes AR, O'Brien K, Chiang M, Skarsgard ED, Chiu PP, et al. Multi-institutional follow-up of patients with congenital diaphragmatic hernia reveals severe $d$

Recebido em 03/10/2012

Aceito para publicação em 06/11/2012

Conflito de interesse: nenhum

Fonte de financiamento: nenhuma

\section{Como citar este artigo:}

Catre D, Lopes MF, Madrigal A, Oliveiros B, Cabrita AS, Viana JS, Neves JF. Fatores preditivos de complicações graves em cirurgia neonatal. Rev Col Bras Cir. [periódico na Internet] 2013;40(5). Disponível em URL: http://www.scielo.br/rcbc

\section{Endereço para correspondência:}

Maria Francelina Lopes

E-mail: mfrancelina@yahoo.com 